\title{
SPECTROSCOPIC AND MOLECULAR DOCKING STUDIES ON THE INTERACTION OF DIMETRIDAZOLE WITH HUMAN SERUM ALBUMIN
}

\author{
WANJU ZHANG ${ }^{1,21^{*}}$, FANG WANG $^{1}$, XUJIE XIONG ${ }^{1}$, YUSHU GE ${ }^{2}$, YI LIU ${ }^{2}$ \\ ${ }^{I}$ School of Chemical Engineering, Huanggang Normal University, Huanggang, 438000, P. R. China. \\ ${ }^{2}$ State Key Laboratory of Virology \& Key Laboratory of Analytical Chemistry for Biolgy and Medicine (MOE), College of Chemistry and Molecular Sciences, \\ Wuhan University, Wuhan 430072, P. R. China. \\ (Received: August 7, 2012 - Accepted: March 8, 2013)
}

\begin{abstract}
Dimetridazole (DMZ) is widely used for the therapeutic treatment of farmed animals and is suspected of being human carcinogens and mutagens. The interaction between DMZ and human serum albumin (HSA) was investigated systematically by fluorescence spectroscopy, synchronous fluorescence, threedimensional fluorescence, CD spectroscopy, UV-vis absorption spectroscopy and molecular docking study. The results indicated that the probable quenching mechanism of HSA by DMZ was dynamic quenching. The corresponding thermodynamic parameters $\Delta H, \Delta S$ and $\Delta G$ were calculated according to Van't Hoff equation. The results $\left(\Delta H=65.10 \mathrm{~kJ} \mathrm{~mol}^{-1}\right.$ and $\left.\Delta S=295.65 \mathrm{~J} \mathrm{~mol}^{-1} \mathrm{~K}^{-1}\right)$ indicated that the driving force between DMZ and HSA was mainly typical hydrophobic interaction. The conformation changes in the interaction were studied by synchronous fluorescence, CD spectroscopy and three-dimensional fluorescence spectra. The results revealed that the microenvironment and conformation of HSA has been changed. Molecular modeling study further confirmed the binding mode obtained by experimental study.
\end{abstract}

Keywords: dimetridazole, human serum albumin, fluorescence quenching, molecular docking, interaction.

\section{INTRODUCTION}

Dimetridazole (DMZ, 1,2-dimethyl-5-nitroimidazole) is a nitroimidazole drug used in veterinary medicine to prevent and treat histomoniasis and coccidiosis in poultry and game birds'. It has also been used for the treatment of genital trichomoniasis in cattle and haemorrhagic enteritis in pigs. The use of DMZ in veterinary practice is strictly regulated in many countries because of DMZ and its metabolites are suspected of being human carcinogens and mutagens ${ }^{2-5}$. However, the chemical-grade powder of DMZ is available and many pharmacies can compound the drug into tablets or capsules ${ }^{6,7}$. The residue of DMZ is often found in food producing animals or in products intended for human consumption. Therefore, studies on the interaction between DMZ and human serum albumin (HSA) are of fundamental importance for providing more information about the potential toxicological effect of DMZ.

Human serum albumin (HSA) is a principal extracellular protein constituent of blood plasma. It plays a dominant role in the transport and disposition of a variety of endogenous and exogenous substances such as drugs. The primary pharmacokinetics function of HSA is participating in the absorption, distribution, metabolism and excretion of drug. It was reported that most drugs travel in plasma and reach the target tissues by binding to HSA $^{8}$. This means that the drug distribution is somewhat controlled by HSA. So study on the drug-protein interaction can assume significance to understand the pharmacokinetic behavior of a drug thoroughly.

In this paper, the interaction of DMZ with HSA was systemically studied by fluorescence spectroscopy, synchronous fluorescence, three-dimensional fluorescence, CD spectroscopy, UV-vis absorption spectroscopy and molecular docking study under simulative physiological condition. The results of this work contribute to understanding the biological effect of DMZ.

\section{EXPERIMENTAL}

\subsection{Materials}

DMZ was obtained from Huanggang Huayang Pharmaceutical Co., Ltd. HSA $(99 \%)$ was purchased from Sigma-Aldrich Inc., and used without further purification. HSA solution was prepared on the basis of its molecular weight of 67,000 and kept in a refrigerator at $4 \square$ and dissolved in PBS (pH 7.4) at the concentration of $1 \times 10^{-5} \mathrm{~mol} \mathrm{~L}^{-1}$. All the other starting materials used were analytical grade. Water used in all procedures was prepared using a Millipore water purification system.

2.2 Fluorescence spectral measurements

All fluorescence spectra were recorded with a LS-55 spectrofluorimeter (Perkin-Elmer corporate, UK) equipped with quartz cells $(1.0 \mathrm{~cm})$ and a thermostat bath. The fluorescence emission spectra were performed at different temperatures $(298,303,308$, and $313 \mathrm{~K})$. An excitation wavelength of 280 $\mathrm{nm}$ was used throughout to minimize the contribution of the tyrosine residues to the emission. The slit widths for excitation and emission were set to 15.0 and $12.0 \mathrm{~nm}$, respectively. The three-dimensional fluorescence spectra were excited at $200 \mathrm{~nm}$ and measured with the emission wavelength from 200 to $500 \mathrm{~nm}$. The number of scanning was 31 at the increment at $5 \mathrm{~nm}$ per scanning.

2.3 UV-vis absorption spectral measurements

TU-1901 spectrophotometer (Puxi Ltd. of Beijing, China) was used to scan UV-vis spectra. The UV-visible absorption spectra were measured with a $1 \mathrm{~cm}$ quartz cell. The concentration of HSA and DMZ were $1 \times 10^{-5} \mathrm{~mol} \mathrm{~L}^{-1}$ and the absorption spectra were recorded from 250 to $450 \mathrm{~nm}$ at room temperature.

2.4 CD measurements

CD measurements were carried out on a Cirsular Dichroism Photomultiplier from Applied Photophysics Limited, UK, using a cylindrical with $0.1 \mathrm{~cm}$ path length. In the experiment, the concentration of HSA was kept at $2.0 \times 10^{-6} \mathrm{~mol}$ $\mathrm{L}^{-1}$. The spectra were recorded in the absence and presence of DMZ with the DMZ/HSA ratio 0:1, 2:1, 10:1 and 30:1, respectively. The CD profiles were obtained by employing a scan speed of $500 \mathrm{~nm} \mathrm{~min}^{-1}$ and a response time of 0.5 s. The spectra were recorded from 200 to $260 \mathrm{~nm}$ at room temperature under constant nitrogen flush.

2.5 Molecular docking investigation

The structure of DMZ was generated by Sybyl 8.1 package. The molecule was charged using Gasteiger and Marsili method, and then optimized using Tripos Force Field.

The crystal structure of HSA was obtained from RCSB Protein Database Bank (PDB code: 1H9Z, 2BXG chain a), the structure of the biopolymer was analyzed and prepared for the docking experiment. $\mathrm{H}$ atoms with $\mathrm{H}$-bond orientation were added. All the ligands and water molecules were removed, the relevant side-chains were repaired and the bumps were fixed, and finally the biopolymer was charged using AMBER7 FF99 method. This final structure was used for the Surflex-Dock program, to compute the binding mode of the compounds with HSA.

Docking study of the binding mode between DMZ and HSA was conducted by Surflex-Dock program of Sybyl 8.1 software package. For HSA, two binding sites were protomoled by choosing the corresponding ligands (WAR in 1H9Z, IBP in 2BXG chain a). The additional starting conformations per molecule was set as 7 for DMZ. Angstroms to expand search grid were set to 6 and the density of search was 3.00. Ring flexibility was considered and the grid was treated softly during the docking study. The results of the binding mode were analyzed and the graphs were mapped with the same program.

\section{RESULTS AND DISCUSSION}

3.1 Fluorescence measurements

In this experiment, the HSA solution concentration was stabilized at 
$1 \times 10^{-5} \mathrm{~mol} \mathrm{~L}^{-1}$, and the concentrations of DMZ were varied from 0 to $2.5 \times 10^{-5}$ mol L ${ }^{-1}$ at increments of $0.25 \times 10^{-5} \mathrm{~mol} \mathrm{~L}^{-1}$. The fluorescence spectra of HSA obtained in the presence of increasing amounts of DMZ are shown in Fig. 1. When excited with a wavelength of $280 \mathrm{~nm}$, HSA has a strong fluorescence emission peaked at $342 \mathrm{~nm}$. The addition of DMZ caused a gradual decrease in the fluorescence intensity of HSA and the emission maximum had a red-shift of $7 \mathrm{~nm}$. This suggested that the microenvironment around HSA was changed after the addition of $\mathrm{DMZ}^{9}$

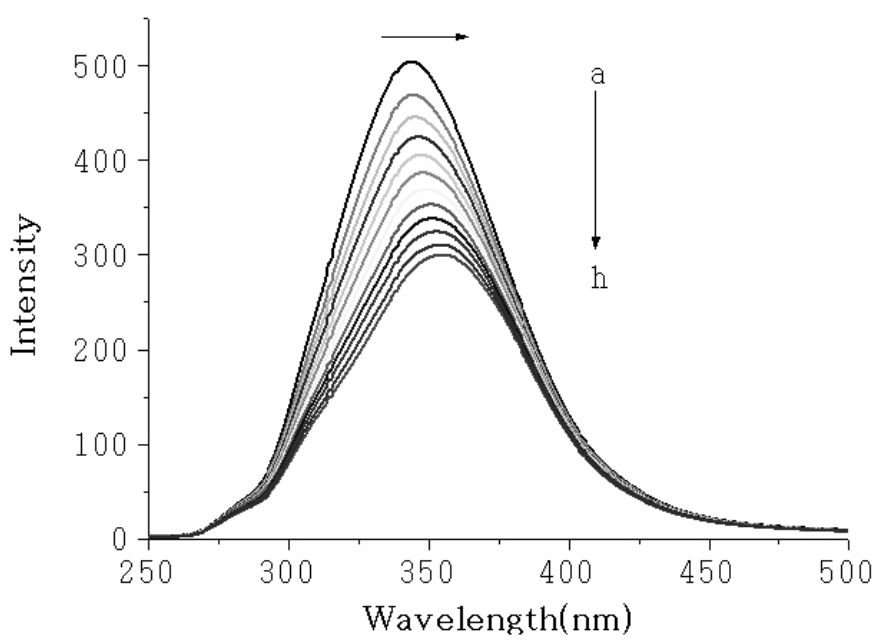

Fig. 1. Emission spectra of HSA in the presence of various concentrations of DMZ $(\mathrm{T}=298 \mathrm{~K} ; \lambda=280 \mathrm{~nm}) . \mathrm{c}(\mathrm{HSA})=1.0 \times 10^{-5} \mathrm{~mol} \mathrm{~L}^{-1} ; \mathrm{c}(\mathrm{DMZ}) /\left(10^{-5}\right.$ mol L $\left.{ }^{-1}\right)$ a-h: $0 ; 0.25 ; 0.5 ; 0.75 ; 1.0 ; 1.25 ; 1.5 ; 1.75 ; 2.0,2.25 ; 2.5$, respectively.

To study the quenching process by DMZ more closely, fluorescence tests were performed at four different temperatures $(298 \mathrm{~K}, 303 \mathrm{~K}, 308 \mathrm{~K}$ and $313 \mathrm{~K}$, respectively). The two mechanisms of quenching, namely dynamic quenching and static quenching, can be distinguished by their differing dependence on temperature and viscosity. Dynamic quenching depends upon diffusion. Since higher temperature results in larger diffusion coefficient, the bimolecular quenching constant is expected to be higher with increasing temperature. In contrast, increased temperature is likely to result in decreased stability of the complex, and thus lower value of the static quenching constant. The possible quenching mechanism was analyzed by the Stern-Volmer equation ${ }^{10}$ :

$$
\frac{F_{0}}{F}=1+k_{\mathrm{q}} \tau_{0}[Q]=1+K_{\mathrm{sV}}[Q]
$$

where $F_{0}$ and $F$ are the steady-state fluorescence intensities in the absence and presence of quencher (DMZ), respectively, $k$ the bimolecular quenching rate constant, $\tau_{0}$ the life time of fluorescence in absence of quencher, $K_{\mathrm{s}}$ the Stern-Volmer quenching constant, and $[Q]$ the concentration of quencher. Hence the above equation could be applied to determine $K_{\mathrm{SV}}$ by linear regression of a plot of $F_{0} / F$ against $[Q]$. Fig. 2 displays the Stern-Volmer plots of the fluorescence quenching of HSA by DMZ at different temperatures. The result shows that the quenching constant (Fig. 2 and Table 1), $K_{\mathrm{SY}}$, increases with increasing temperature. This indicates that the interactions between DMZ and HSA are initiated by dynamic collision rather than by compound formation.

Table 1. Stern-Volmer quenching constants for the interaction of DMZ with HSA at four different temperatures.

\begin{tabular}{|c|c|c|c|c|c|}
\hline $\mathrm{pH}$ & $T(\mathrm{~K})$ & $K_{\mathrm{SV}}\left(\times 10^{4} \mathrm{M}^{-1}\right)$ & $k_{\mathrm{q}}\left(\times 10^{12} \mathrm{M}^{-1} \mathrm{~s}^{-1}\right)$ & $R^{\mathrm{a}}$ & S.D. $^{\mathrm{b}}$ \\
\hline \multirow{4}{*}{7.4} & 298 & 2.440 & 2.440 & 0.999 & 0.0023 \\
\cline { 2 - 6 } & 303 & 2.507 & 2.507 & 0.999 & 0.0025 \\
\cline { 2 - 6 } & 308 & 2.576 & 2.576 & 0.998 & 0.0029 \\
\cline { 2 - 6 } & 313 & 2.615 & 2.615 & 0.999 & 0.0021 \\
\hline
\end{tabular}

a Linear correlated coefficient.

${ }^{\mathrm{b}}$ Standard deviation for the $K_{\mathrm{Sv}}$ values.

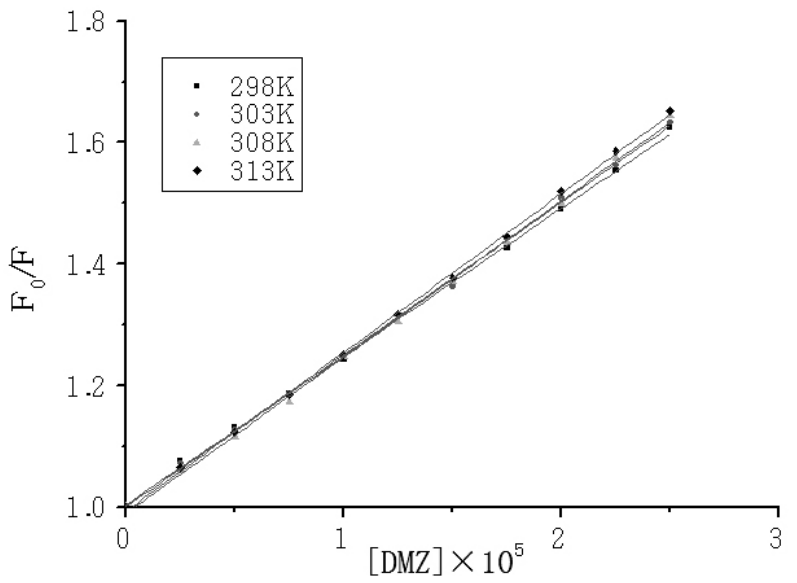

Fig. 2. Stern-Volmer plots for the quenching of HSA by DMZ at the four different temperatures, $\mathrm{pH}=7.4$.

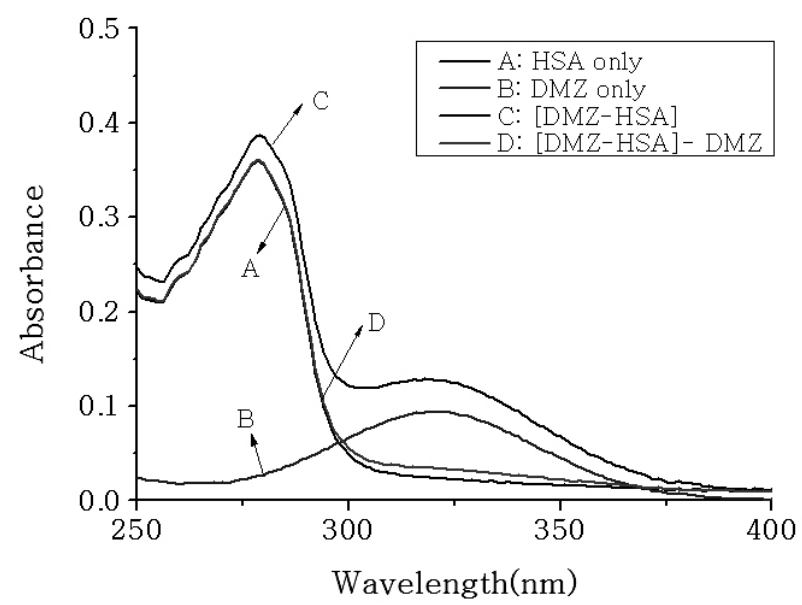

Fig. 3. UV-vis absorption spectra of HSA, DMZ and DMZ-HSA solutions. $\mathrm{c}(\mathrm{HSA})=\mathrm{c}(\mathrm{DMZ})=1.0 \times 10-5 \mathrm{~mol} \mathrm{~L}-1$. (A) The absorption spectrum of HSA only; (B) the absorption spectrum of DMZ only; (C) the absorption spectrum of compound DMZ-HSA when the mole ratio is $1: 1$; (D) the difference absorption spectrum between DMZ-HSA and DMZ at the same concentration.

UV-vis absorption spectra experiment is performed to identify the real mechanism of this quenching procedure. UV-vis absorption measurement is a very simple method to know the complex information and usually applied to explore the structural change. Dynamic quenching only affects the excited states of the fluorophores, and there is no change in the absorption spectra. To reconfirm the quenching mechanism, we used the difference absorption spectroscopy to obtain spectra, the UV-vis absorption spectra of HSA and the difference in the absorption spectra of DMZ-HSA and DMZ at the same concentrations. As can be seen from Fig. 3, the UV absorption spectrum of HSA (Fig. 3, curve A) and the absorption spectrum (Fig. 3, curve D) by subtracting the absorption spectrum of DMZ from that of DMZ-HSA at the same concentration could be overlapped within experimental error. This result reconfirms that the probable quenching mechanism of fluorescence of HSA by DMZ is a dynamic quenching procedure ${ }^{11}$.

\subsection{Binding constant and binding site numbers}

When small molecules bind independently to a set of equivalent sites on amacromolecule, the equilibrium between free and bound molecules is given by the equation ${ }^{12}$ :

$$
\log \left(\frac{F_{0}-F}{F}\right)=\log K_{\mathrm{b}}+n \log [Q]
$$




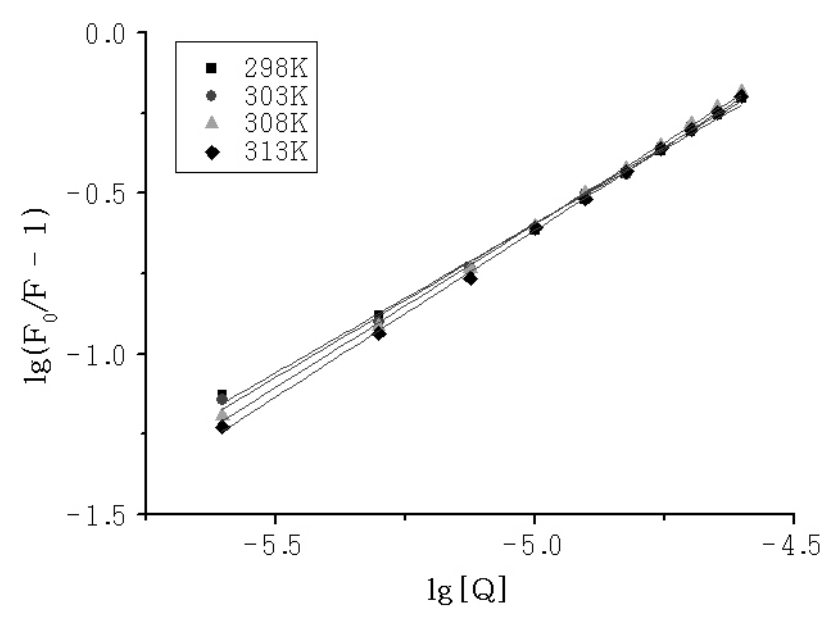

Fig. 4. Double-log plots of DMZ quenching effect on HSA fluorescence at different temperatures.

where $K_{\mathrm{b}}$ is the observed binding constant to a site and $n$ is the number of binding sites per HSA. The dependence of $\log \left(F_{0} / F-1\right)$ on the value of $\log [Q]$ is linear with the slope equal to the value of $n$ and $\log K_{\mathrm{b}}$ is fixed on the ordinate. Fig. 4 is the plots of $\log \left(F_{0} / F-1\right)$ versus $\log [Q]$ for the DMZ-HSA system at different temperatures. The binding constants $K_{\mathrm{b}}$ and binding sites $n$ were listed

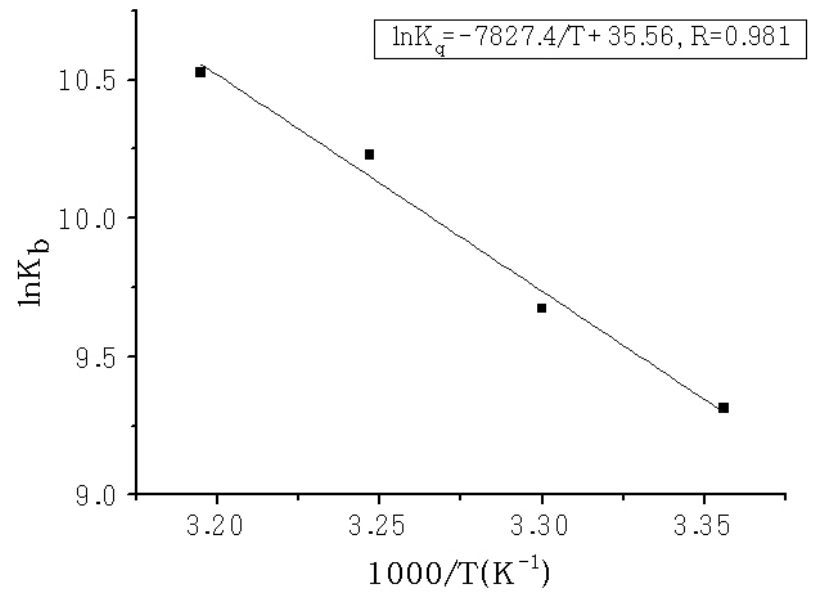

Fig. 5. Arrhenius plot for interaction between HSA and DMZ.

in Table 2. We can see from the table that the values of $K_{\mathrm{b}}$ increased with the rising temperatures, which indicated that the binding is endothermic reaction ${ }^{13}$, and the capacity of DMZ binding to HSA is enhanced with the increasing temperature. The values of $n$ approximately equal to 1 , which indicated the existence of just one single binding site in HSA for DMZ.

Table 2. Apparent binding constants $K_{\mathrm{b}}$ at different temperatures and relative thermodynamic parameters of the DMZ-HSA system.

\begin{tabular}{|c|c|c|c|c|c|c|c|c|}
\hline$p H$ & $T(K)$ & $K_{b}\left(\times 10^{4} M^{-1}\right)$ & $n$ & $R^{a}$ & S.D. ${ }^{b}$ & $\Delta H\left(\mathrm{~kJ} \mathrm{~mol}^{-1}\right)$ & $\Delta G\left(\mathrm{~kJ} \mathrm{~mol}^{-1}\right)$ & $\Delta S\left(\mathrm{~J} \mathrm{~mol}^{-1} \mathrm{~K}^{-1}\right)$ \\
\hline \multirow{3}{*}{7.4} & 298 & 1.1058 & 0.9273 & 0.997 & 0.0176 & \multirow{3}{*}{65.10} & -23.03 & \multirow{3}{*}{295.65} \\
\hline & 303 & 1.5889 & 0.9590 & 0.997 & 0.0173 & & -24.53 & \\
\hline & 308 & 3.0563 & 1.0163 & 0.999 & 0.0099 & & -26.04 & \\
\hline
\end{tabular}

${ }^{\mathrm{a}}$ Linear correlated coefficient for the $K_{\mathrm{b}}$ values.

${ }^{\mathrm{b}}$ Standard deviation for the $K_{\mathrm{b}}$ values.

\subsection{Type of interaction force between DMZ and HSA}

Generally speaking, the force between small organic molecule and biological macromolecule includes hydrophobic force, hydrogen bond, van der Waals force and electrostatic interactions, etc. ${ }^{14}$ The thermodynamic parameters, enthalpy change $(\Delta H)$ and entropy change $(\Delta S)$ of the reaction are important for the study of the interaction force. For this reason, the temperaturedependent binding constant was studied.

The values of $\Delta H$ and entropy change $\Delta S$ can be estimated from the following van't Hoff equation:

$$
\ln K=-\frac{\Delta H}{R T}+\frac{\Delta S}{R}
$$

and the free energy changes $(\Delta G)$ at different temperatures can be calculated from the following relationship:

$$
\Delta G=\Delta H-T \Delta S=-R T \ln K
$$

In Eq. (3), $K$ is analogous to the effective quenching constants $K_{\mathrm{b}}$ at the corresponding temperature. If the temperature only changes a little, the enthalpy change $(\Delta H)$ can be regarded as a constant and it can be calculated from the slope of the van't Hoff relationship.

The thermodynamic parameters were calculated from Eqs. (3) and (4) (Fig. 5) and summarized in Table 2. The values of enthalpy change $(\Delta H)$ and entropy change $(\Delta S)$ were found to be $65.10 \mathrm{~kJ} \mathrm{~mol}^{-1}$ and $295.65 \mathrm{~J} \mathrm{~mol}^{-1}$ $\mathrm{K}^{-1}$, which indicated that the driving force of this process was mainly typical hydrophobic interaction $^{15,16}$, and hydrogen bonds and van der Waals force were not dominant. The negative value of $\Delta G^{\ominus}$ reveals that the interaction process between DMZ and HSA is spontaneous.
3.4 Conformation investigations

3.4.1 Synchronous fluorescence spectroscopy studies

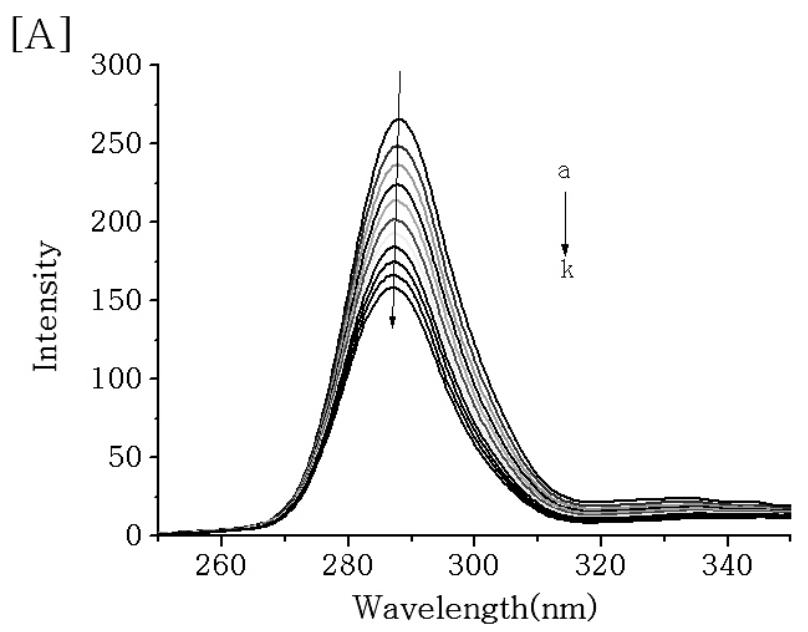




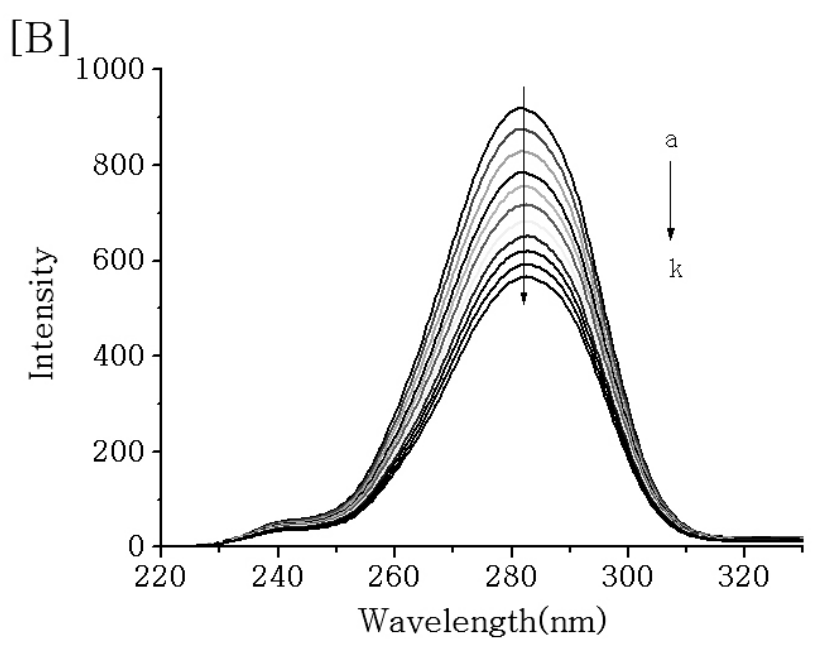

Fig. 6. Synchronous fluorescence spectra of HSA: (a) $\Delta \lambda=15 \mathrm{~nm}$; (b) $\Delta \lambda$ $=60 \mathrm{~nm} . \mathrm{c}(\mathrm{HSA})=1.0 \times 10-5 \mathrm{~mol} \mathrm{~L}-1 ; \mathrm{c}(\mathrm{DMZ}) /(10-5 \mathrm{~mol} \mathrm{~L}-1), \mathrm{a}-\mathrm{k}: 0 ; 0.25$; $0.5 ; 0.75 ; 1.0 ; 1.25 ; 1.5 ; 1.75 ; 2.0,2.25 ; 2.5$, respectively.

The synchronous fluorescence spectroscopy introduced by Llody ${ }^{17}$ is a very useful method to study the microenvironment of amino acid residues by measuring the emission wavelength shift $^{18}$. When the $D$-value $(\Delta \lambda)$ between excitation and emission wavelength are stabilized at 15 or $60 \mathrm{~nm}$, the synchronous fluorescence gives the characteristic information of tyrosine or tryptophan residues, respectively ${ }^{19}$. As shown in Fig. $6 \mathrm{~A}$, when $\Delta \lambda$ was set to $15 \mathrm{~nm}$, the maximum emission wavelength has a slight blue shift at the investigated concentrations range. This result indicated that the conformation of HSA was changed and it suggested a less polar (or more hydrophobic) environment of tyrosine residue. While when $\Delta \lambda=60 \mathrm{~nm}$ (Fig. 6B), the maximum emission wavelength kept the position, which revealed that there was no change of the microenvironment of the tryptophan residue. Moreover, the fluorescence intensity decreased regularly with the addition of DMZ in both figures. This result further demonstrated the occurrence of fluorescence quenching in the DMZ-HSA binding process.

3.4.2 Circular dichroism spectra

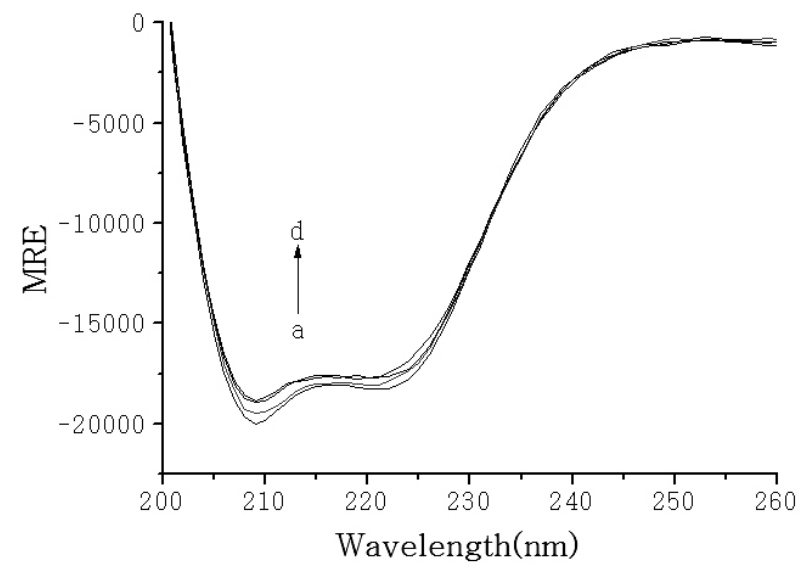

Fig. 7. The far-UV CD spectra of the DMZ-HSA system obtained in PBS of $\mathrm{pH} 7.4$ at room temperature. $\mathrm{c}(\mathrm{HSA})=2.0 \times 10-6 \mathrm{~mol} \mathrm{~L}-1 ; \mathrm{c}(\mathrm{DMZ}) /(10-6$ mol L-1): a-d: $0 ; 2.0 ; 10.0 ; 30.0$, respectively.

Table 3. Parameters of CD Spectral Study of DMZ-HSA System.

\begin{tabular}{|c|c|c|c|}
\hline$H S A / D M Z$ & $\alpha$-helix & $\beta$-strand & unordered \\
\hline $1: 0$ & 0.596 & 0.062 & 0.235 \\
\hline $1: 2$ & 0.588 & 0.064 & 0.239 \\
\hline $1: 10$ & 0.568 & 0.070 & 0.246 \\
\hline $1: 30$ & 0.554 & 0.072 & 0.246 \\
\hline
\end{tabular}

The CD spectra of HSA with various concentration of DMZ in PBS ( $\mathrm{pH}=$ 7.4) and at room temperatures are shown in Fig. 7. It can be seen from the figure that HSA exhibits two negative bands at 208 and $222 \mathrm{~nm}$, which representes the typical $\alpha$-helix structure of protein. The CD results were expressed in terms of mean residue ellipticity (MRE) in deg $\mathrm{cm}^{2} \mathrm{dmol}^{-1}$ according to the following equation:

$$
\mathrm{MRE}=\frac{\text { observed } \mathrm{CD}(\mathrm{mdeg})}{\mathrm{C}_{\mathrm{p}} \mathrm{nl} \times 10}
$$

where $C$ is the molar concentration of the protein, $n$ the number of amino acid residues (585 for HSA) and $l$ the path-length $(0.1 \mathrm{~cm})$. The $\alpha$-helical contents of free and combined HSA were calculated from MRE values at 208 $\mathrm{nm}$ using the equation:

$$
\alpha-\operatorname{helix}(\%)=\frac{-\mathrm{MRE}_{208}-4000}{33000-4000}
$$

where $\mathrm{MRE}_{208}$ is the observed MRE value at $208 \mathrm{~nm}, 4000$ is the MRE of the $\beta$-form and random coil conformation cross at $208 \mathrm{~nm}$, and 33,000 is the MRE value of a pure $\alpha$-helix at $208 \mathrm{~nm}$. From the above equation, the $\alpha$-helicity in the secondary structure of HSA was determined. The contents of $\alpha$-helix and $\beta$-strand are calculated using SELCON3. The results are listed in Table 3. The decrease in the $\alpha$-helix content and the increase in the $\beta$-strand content suggest that DMZ destroys the natural structure of $\mathrm{HSA}^{20}$.

3.4.3 Three-dimensional fluorescence spectra
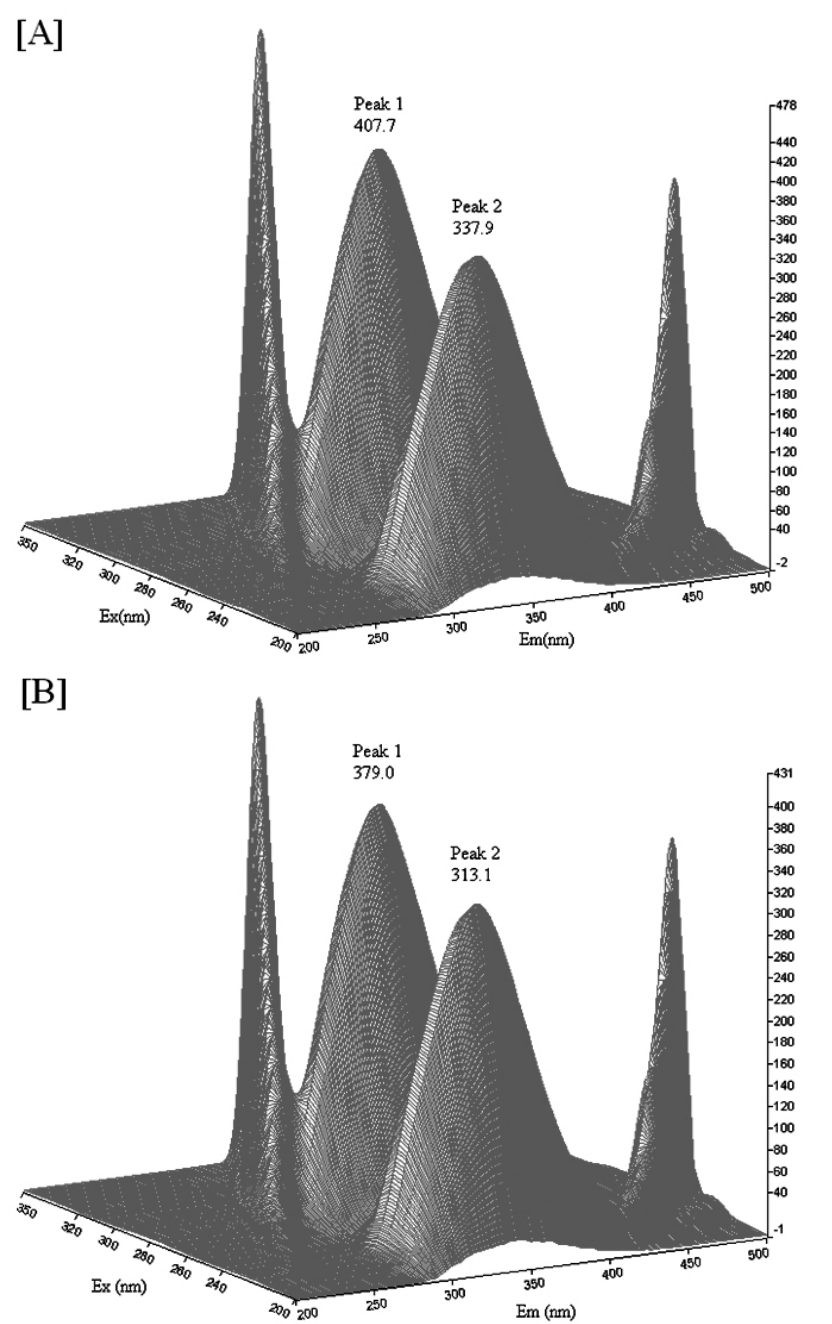

Fig. 8. Three-dimensional fluorescence spectra of HSA and DMZ-HSA system. $\mathrm{c}(\mathrm{HSA}) /(10-6 \mathrm{~mol} \mathrm{~L}-1)$ : A: $2.0 ; \mathrm{B}: 2.0 ; \mathrm{c}(\mathrm{DMZ}) /(10-6 \mathrm{~mol} \mathrm{~L}-1)$ : A: $0 ;$ B: 2.0 . 
The three-dimensional fluorescence spectra were also applied to monitor the changes in the secondary structure of protein and their dynamics. Fig. 8 shows the three-dimensional fluorescence spectra of HSA (Fig. 8A) and DMZ-HSA system (Fig. 8B), respectively. Two peak regions (peak 1 at 280 $\mathrm{nm} / 345 \mathrm{~nm}$ and peak 2 at $225 \mathrm{~nm} / 343 \mathrm{~nm}$ ) were observed. Peak 1 shows the spectral characteristics of tryptophan and tyrosine residues. Peak 2 relates to the fluorescence spectral behavior of polypeptide backbone structures, and its intensity is correlated with the secondary structure. From Fig. 8 we can see that both of fluorescence peaks in the three-dimensional fluorescence spectra of HSA are quenched. This result indicates the interaction of DMZ with HSA has induced some micro-environmental and conformational changes of $\mathrm{HSA}^{21}$.

On the basis of the studies of synchronous fluorescence spectra, CD spectra and 3D fluorescence spectra, it is concluded that the interaction of DMZ with HSA has induced some slight conformational changes of the protein.

\subsection{Molecular Docking Study}
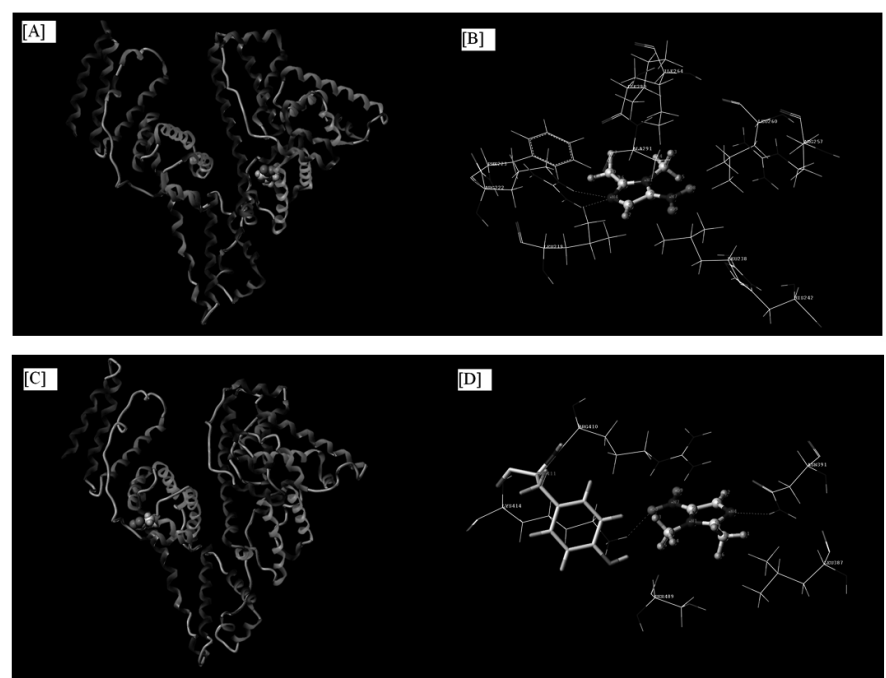

Fig. 9. The binding mode between DMZ and HSA. [A] and [B]: Detail in site I, [C] and [D]: Detail in site II. The inserts are the locations of DMZ binding on HSA. The residues of HSA are represented by solid lines and DMZ by ball and stick for clarity. The hydrogen bonds between DMZ and HSA are represented by yellow dashed lines.

Molecular modeling method has been employed to promote the understanding of the interactions between drugs and $\mathrm{HSA}^{22}$. The descriptions of 3D structure of crystalline albumin have revealed that HSA comprises of three homologous domains that assemble to form a heart-shaped molecule ${ }^{23}$. HSA is monomeric but contains the three structurally similar $\alpha$-helical domains (I-III); each domain has two subdomains (A and B), which are six (A) and four (B) $\alpha$-helices, respectively. The principal regions of ligand binding to HSA are located in hydrophobic cavities in subdomains IIA and IIIA, i.e. sites I and II, respectively. Studies have shown that HSA can bind many ligands in several binding sites ${ }^{24}$.

To further understand the interaction of DMZ and HSA, we have run a docking program to simulate the binding mode between DMZ and HSA and the result is shown in Fig. 9. From the figure we can see that DMZ molecule is buried within hydrophobic cavities in sub-domain IIA (Sudlow's site I) and sub-domain IIIA (Sublow's site II). This finding provides a good structural basis to explain the efficient fluorescence quenching of HSA in the presence of DMZ. The docking scores (stand for $\mathrm{p} K$ ) for site I and site II are 2.97 and 3.20 , respectively. Since higher score indicates lower dissociation constant $K_{\mathrm{d}}$, we can conclude that the prior binding site for DMZ is site II. The study shows that DMZ interacting with Tyr411 in site II, which dominates the fluorescence quenching of HSA. The interaction between DMZ and HSA was dominated by hydrophobic contacts ${ }^{21}$. Furthermore, hydrogen bonds are also observed between DMZ and the residues of HSA, which can increase the stability of DMZ-HSA system ${ }^{25}$. The result of molecular modeling study is in good accordance with the experimental study.

\section{CONCLUSION}

The interaction between DMZ and HSA was studied by fluorescence spectroscopy combined with UV-vis and CD spectroscopy. The results indicated that the fluorescence quenching mechanism for HSA through DMZ binding was likely a dynamic quenching. The binding reaction of DMZ with HSA was spontaneous and mainly mediated by typical hydrophobic interaction. The experimental results also showed that the binding of DMZ to HSA can induce conformational changes to HSA. Such conformation changes could induce toxic effects. Thus, this paper provides a new strategy to explore the biological interaction of DMZ with HSA at molecular level.

\section{ACKNOWLEDGEMENT}

This work was financially supported by the National Science Fund for Distinguished Young Scholars of China (Grant No: 21225313), and the Chinese 973 Proram (No: 2011CB933600)

\section{REFERENCES}

E. Daeseleire, H. D. Ruyck, R. V. Renterghem, Analyst. 125, 1533, (2000) C. E. Voogd, Mutat. Res. 86, 243, (1981)

L. Dobiás, M. Cerná, P. Rössner, R. Srám, Mutat. Res. 317, 177, (1994)

W. Raether, H. Hänel, Parasitol. Res. 90, S19, (2003)

C. E. Voogd, J. J. van der Stel, J. J. Jacobs, Mutat. Res. 26, 483, (1974)

M. K. Tolbert, J. Gookin, Compend. Contin. Educ. Vet. 31, 374, (2009)

T. W. Rosado, A. Specht, S. L. Marks, J. Vet. Intern. Med. 21, 328, (2007)

T. Peters, Adv. Protein. Chem. 37, 161, (1985)

T. Yuan, A. M. Weljie, H. J. Vogel, Biochem. 37, 3187, (1998)

10 J. R. Lakowicz, Principles of Fluorescence Spectroscopy, 3rd ed., Plenum Press, New York, 2006; pp. 277-285

11 E. Froehlich, J. S. Mandeville, C. J. Jennings, R. Sedaghat-Herati, H. A. Tajmir-Riahi, J. Phys. Chem. B 113, 6986, (2009)

12 M. X. Xie, M. Long, Y. Liu, C. Qin, Y.D. Wang, Biochim. Biophys. Acta. $1760,1184,(2006)$

13 Y. Q. Wang, H. M. Zhang, G. C. Zhang, W. H. Tao, S. H. Tang, J. Mol. Struct. 830, 40, (2007)

14 D. Leckband, Annu. Rev. Biophys. Biomol. Struct. 29, 1, (2000)

15 P. D. Ross, S. Subramanian, Biochem. 20, 3096, (1981)

16 Timaseff, Thermodynamics of protein interactions, in: H. Peeters (Ed.), Proteins of biological fluids, Pergamon Press, Oxford, 1972; pp. 511-519

17 J. B. F. Llody, Nat. Phys. Sci. 231, 64, (1971)

18 W. C. Abert, W. M. Gregory, G. S. Allan, Anal. Biochem. 213, 407, (1993)

19 J. N. Miller, Proc. Anal. Div. Chem. Soc. 16, 203, (1979)

20 F. F. Tian, F. L. Jiang, X. L. Han, C. Xiang, Y. S. Ge, J. H. Li, Y. Zhang, R. Li, X. L. Ding, Y. Liu, J. Phys. Chem. B 114, 14842, (2010)

21 Y. Sun, Y. Y. Zhao, G. B. Li, J. Chin. Chim. Soc. 58, 602, (2011)

22 P. Miskovsky, J. Hritz, S. S. Cortes, G. Fabriciova, J. Ulicny, L. Chinsky, J. Photochem. Photobiol. 74, 172, (2001)

23 A. A. Bhattacharya, S. Curry, N. P. Franks, J. Biol. Chem. 275, 38731 , (2000)

24 J. Ghuman, P. A. Zunszain, I. Petitpas, A. A. Bhattacharya, M. Otagiri, S. Curry, J. Mol. Biol. 353, 38, (2005)

25 J. X .Fu, Y. S. Ge, F. L. Jiang, X. H. Sun, Y. Liu, Sci. China. ser B 54, $788,(2011)$ 